\title{
Empresa y privacidad: el cuidado de la información y los datos personales en medios digitales*
}

\section{Ana lsabel Meraz Espinoza**}

\begin{abstract}
RESUMEN
La tecnología como tema de investigación es amplio. Si se enfoca en aspectos propios de una empresa se abre un abanico ilimitado de asuntos de carácter legal y técnico que requieren de atención y cuidado ante sus posibles consecuencias juridicas. La moderna empresa, inserta en la sociedad de la información y del conocimiento, cambia y evoluciona de manera constante y se adapta a ese vaivén técnico y legal que obligatoriamente le sujeta. Un asunto importante al que se enfrentan todo tipo de corporaciones, pequeñas, medianas o grandes, es el uso cotidiano de los medios digitales para procesar, distribuir o almacenar cualquier tipo de información en algún dispositivo electrónico. Esta actividad, tan necesaria en la actualidad, sigue facilitándole al empresario todo tipo de procesos. Sin embargo, la empresa es vulnerable ante los riesgos técnicos y legales relativos a la protección de la privacidad de la información y datos personales de quienes la integran. La privacidad comprende una serie de situaciones relacionadas con el estatus personal y particular de cada individuo, por ello es importante analizar algunas de ellas para determinar hasta donde llegan los derechos y las obligaciones que atañen a cada una de las partes involucradas en un contexto empresarial.
\end{abstract}

\section{PALABRAS CLAVE}

Derecho, privacidad, empresa, información, datos personales, medios digitales.

\begin{abstract}
Technology as a research topic is broad. Focusing on aspects of a business unlocks an unlimited range of legal and technical issues that require care and attention to its possible legal consequences. The modern company, inserted in the society of information and knowledge, constantly changes and evolves and adapts to that technical and legal swing that inevitably subjects it. An important issue faced by all types of corporations, small, medium or large, is the daily use of digital media to process, distribute or store any kind of information in an electronic device. This activity, so necessary at present, continues to facilitate the entrepreneur all kinds of processes. However, the company is vulnerable to technical and legal risks related to the protection of privacy of information and personal data of its members. Privacy involves a series of situations related to the personal and individual status of each individual, so it is important to analyze some of them to determine the extent to which the rights and obligations that affect each of the parties involved in a business context.
\end{abstract}

\section{KEYWORDS}

Law, Privacy, Company, Information, Personal Data, Digital Media.

\footnotetext{
*Artículo recibido el 6 de junio de 2017 y aceptado el 10 de octubre de 2017

**Tecnológico de Monterrey, Escuela de Ciencias Sociales y Gobierno. (ameraz@itesm.mx) orcid 0000-0002-9654-8737
} 
SUM ARIO

1. INTRODUCCIÓN

2. Hacia un significado común de privacidad

3. Implicaciones de un derecho a la privacidad en la empresa

4. Protección de la información y resguardo de datos en la empresa

5. Conclusiones

\section{Introducción}

El derecho a la privacidad en el contexto de los medios digitales, sobre todo a la información considerada como datos personales, es uno de los temas más debatidos en la arena jurídica. El ciberespacio genera, en el día a día, una serie de premisas ante las cuales se debate continua y sistemáticamente. El tema de la privacidad y la protección de los datos personales se ha vuelto un referente obligado en el ámbito empresarial.

Ante el manejo copioso de todo tipo de información, el empresario asume una gran responsabilidad para salvaguardar todo tipo de documentos. La tecnología, tan necesaria para el funcionamiento efectivo de las corporaciones, tiene también un lado sensible que puede ser contraproducente en la efectividad de la organización y de sus procesos cotidianos.

Estos claroscuros de la ciencia de la informática van más allá de la simple operación técnica, debido a que inciden directamente en algunos supuestos de la ciencia jurídica. La privacidad, la información y los datos personales, desde la perspectiva de su sistema operativo, requieren soportes modernos que sean a la vez manejables y seguros.

Desde una perspectiva jurídica, el manejo, uso y almacenamiento de la información necesitan la certeza legal que permita a sus titulares (dueños de la información) confiar en el cuidado de la misma, pero esa certeza es también importante para sus tenedores (poseedores de la información) respecto a su uso y resguardo.

Los temas sobre privacidad, información y datos personales se pueden abordar de manera separada y analizarse de manera particular. Sin embargo, debido a que todos ellos tienen en común la tecnología, hilo conductor que los alinea, y una regulación jurídica especial, se explicarán en este apartado como piezas de un asunto común y estrechamente enlazado.

El impacto que tienen estos temas en la vida cotidiana de los ciudadanos, tanto en sus actividades personales como profesionales, provoca consecuencias 
jurídicas diferentes. Por tanto, cada quién, desde su propia afectación, tiene facultades para demandar las garantías que le correspondan.

Estos problemas se derivan principalmente por el uso inapropiado de los medios electrónicos o errores y negligencias en el manejo de la información que derivan en la comisión de hechos ilícitos. Actualmente, las empresas, pequeñas, medianas o grandes, utilizan la tecnología para todo tipo de procesos, así como una gran cantidad de información que requiere resguardo y protección. Es a partir de esta premisa como se abordará esta temática en el presente análisis.

\section{Hacia un significado común de privacidad}

El término privacidad aplica en todo tipo de actividad, de las personas como entes particulares y a nivel colectivo, como en las tareas que se desempeñan en una corporación. Legalmente, tanto en un caso como en el otro, se tiene como objetivo el preservar los derechos de las personas que, en un actuar individual o colectivo, se vulneren.

En este sentido, con el manejo de la tecnología y el uso de diferentes medios electrónicos, se requiere de mayor cuidado para preservar todo tipo de información. Esto no descarta los soportes físicos que también guardan datos y que, en ambas situaciones, es elemental la custodia. Para el caso de las empresas, es importante destacar la responsabilidad corporativa. En asuntos de privacidad y protección de datos personales, es fundamental la ética por parte de la empresa.

En esta era de la información, las empresas tienen nuevos desafíos "que pueden convertirse en obstáculos o, por el contrario, en oportunidades de crecimiento". ${ }^{1}$ Esto implica ejercer la prudencia, la confianza y la justicia como virtudes básicas. El uso de las nuevas tecnologías de la información y las comunicaciones, con su transitar de datos personales y privados, lo reclaman.

En palabras de Theodore Claypoole y Theresa M. Payton, el término de privacidad, tan complejo como personal, es similar a círculos concéntricos. Estos autores consideran que toda persona está en el centro y, alrededor de ella, existen muy cercanamente otros círculos compuestos por una serie de variables, como sus secretos, pensamientos, rituales y demás asuntos propios que "no comparte con nadie". ${ }^{2}$ Otros autores, como Víctor Drummond, resumen el

\footnotetext{
${ }^{1}$ CoRtina, Adela, Construir confianza. Ética de la empresa en la sociedad de la información y las comunicaciones, Madrid, Trotta, 2003, p. 11.

${ }^{2}$ Claypoole, Ted y Payton, Theresa, Privacy in the Age of Big Data. Recognizing Threats, Defending Your Rights, and Protecting Your Family, Reino Unido, Rowman \& Littlefield, 2014, p. xi.
} 
concepto de privacidad de una manera por demás simple, como "la cómoda distancia que una persona mantiene, espontáneamente, entre su más profunda individualidad y el exterior". ${ }^{3}$

El rol que desempeña el big data en la actualidad, como una muestra en este contexto, es crucial para determinar los tipos de interferencia que existen en los ámbitos de la privacidad en general. La inteligencia de datos, o big data, se define como "la práctica de las compañías para recolectar millones de datos sobre los consumidores y el uso de todos esos datos para predecir tendencias y el desarrollo de mejores ventas y estrategias de mercadotecnia”. ${ }^{4}$ Ésta coloca a los clientes de cualquier establecimiento comercial como proveedores de información íntima y personal, lo cual incidirá directamente en el ánimo de sus decisiones de compra.

Este ejemplo puede dimensionar toda la gama de datos que se proporcionan en los diferentes contextos en que desarrolla sus actividades cualquier ciudadano. No obstante, en el ámbito de los negocios, ocasiona a veces una molestia generalizada ante el acoso y la disposición de información personalísima.

Sin embargo, el tema de la privacidad sigue tomándose de manera subjetiva porque es, ante todo, un asunto individual en donde están involucrados la percepción, los valores, la ética y demás asuntos meramente personales. Aunque la defensa de la privacidad ocupa buena parte de los trabajos sociales y legislativos de la actualidad, algunos autores argumentan que el tema no es nada nuevo. En el artículo "The Right to Privacy", publicado en la Harvard Law Review, número 193 del año de 1890, Samuel D. Warren y Louis Brandeis justificaban la necesidad de regular el derecho a la privacidad o the right to be let alone (el derecho a ser dejado solo). ${ }^{5}$

Ahora, si se toma en cuenta el avance tecnológico y el desarrollo de las tecnologías de información y telecomunicaciones, es apabullante la manera en que la vida íntima de las personas está expuesta a todo tipo de intromisión. Regularmente, cuando se habla de privacidad, se alude a diferentes momentos, como "el pasar tiempo de manera aislada con la familia y amigos. Estar solo con amigos muy cercanos, donde sentimos y actuamos de manera diferente, y estamos con la guardia más baja que cuando estamos en público". ${ }^{6}$

\footnotetext{
${ }^{3}$ Drummond, Victor, Internet, privacidad y datos personales, Madrid, Reus, 2004, p. 149.

${ }^{4}$ Claypoole, Ted y Payton, Theresa, Privacy in the Age of Big Data. Recognizing Threats, Defending Your Rights, and Protecting Your Family, Reino Unido, Rowman \&t Littlefield, 2014, p. 7.

${ }^{5}$ Warren, Samuel y Brandels, Louis, "The Right to Privacy", Harvard Law Review, núm. 193. [Consulta: 20 de febrero, 2017]. Disponible en: http://faculty.uml.edu/sgallagher/Brandeisprivacy.htm

${ }^{6}$ StefiK, Mark, The Internet Edge. Social, Technical, and Legal Challenges for a Networked World. The Digital Keyhole: Privacy Rights and Trusted Systems, Estados Unidos, MIT Press, 2000, p. 199.
} 
En años anteriores, este contexto íntimo era vulnerado principalmente por los medios de comunicación, en contra de la vida personal de figuras públicas. Ahora es diferente, las afectaciones a la intimidad proceden de personas no institucionales, comunes y corrientes, en contra de otras de igual condición y con el único objetivo de hacer pública información de carácter confidencial o privado. ${ }^{7}$

En 1986, se promulgó en Estados Unidos el Electronic Communication Privacy Act (ЕСРA), un documento relativo a la privacidad, cuyas normas prohíben la interceptación de mensajes enviados a través de este tipo de tecnología. Además, el documento define todo lo relacionado a las comunicaciones electrónicas, como el correo electrónico, las transmisiones vía satélite, la telefonía celular, entre otros medios, y establece sanciones civiles y penales cuando se infringen sus normas.

Esta disposición jurídica se aplica para proteger a las personas en asuntos relacionados con el comercio y las finanzas, pero también para otro tipo de actividades de carácter privado, cuando los usuarios emplean los sistemas de comunicación electrónicos y la información que por esos medios circula, a modo de que no pueda ser interceptada y, por lo tanto, no sea intencionalmente captada por terceros, ajenos a los asuntos privados o íntimos que ahí se traten. ${ }^{8}$

Terence Craig y Mary Ludloff dividen en tres tipos básicos la privacidad: privacidad física, la libertad para que no haya intromisiones en lo físico de las personas, posesiones y espacio; información privada, la información personal recolectada en formatos digitales, y privacidad organizacional, la que tienen las agencias de gobierno, compañías y demás organizaciones para no revelar actividades y secretos a personas ajenas. ${ }^{9}$

Por su parte, Mark Stefik menciona tres principales fuentes del derecho de privacidad que existen en los Estados Unidos de América: "Protección constitucional (constitutional protections), el derecho común o jurisprudencia (common law or case law, y el derecho estatutario (statutory law)". ${ }^{10}$ De estas fuentes jurídicas, es el statutory law el que más se aplica en asuntos vinculados con información electrónica. En cuanto a la protección de datos, tanto en los

\footnotetext{
${ }^{7}$ Pinochet, Francisco, Derecho de Internet. Los principios especiales, México, Flores, 2017, p. 113.

8 "Electronic Communication Privacy Act (ECPA)", U. S. Department of Justice, 2016. [Consulta: 27 de marzo, 2017]. Disponible en: https://it.ojp.gov/privacyliberty/authorities/statutes/1285

${ }^{9}$ Craig, Terence y Ludloff, Mary, Privacy and Big Data, Estados Unidos, O'Reilly, 2011, p. 16.

${ }^{10}$ Stefik, Mark, The Internet Edge. Social, Technical, and Legal Challenges for a Networked World. The Digital Keyhole: Privacy Rights and Trusted Systems, Estados Unidos, MIT Press, 2000, p. 201.
} 
Estados Unidos como en la mayoría de los países, se recurre a la legislación formal con nuevas leyes. ${ }^{11}$

Ante la vorágine tecnológica a nivel global y el cada vez más reducido margen de privacidad del cual pueden gozar los ciudadanos con los medios de comunicación electrónicos -que todo lo observan, lo invaden, lo tergiversan y lo divulgan- surgen estas interrogantes: ¿hasta dónde se tiene derecho o se puede permitir que otros individuos entren al mundo personal, familiar, laboral, privado o íntimo de cada persona? ¿Qué leyes aplican a cada caso y qué tipo de sanciones existen?

Probablemente estas preguntas no le importen a buena parte de la población, debido a la fascinación que tiene por la computación, la inmediatez en el intercambio de información con cualquier lugar del planeta o en dar a conocer su vida y, sobre todo, conocer la de los otros. Todo ello se traduce en que la diferencia entre un ciudadano extrovertido y uno introvertido, o cuidadoso de su intimidad, es la cantidad de información que muestra al exterior.

No obstante, en el entorno laboral, donde las empresas poseen y manejan una gran cantidad de información personal de sus empleados, proveedores y demás involucrados en sus procesos de trabajo, hay otras limitantes en el manejo de la privacidad e información personal, por causa de la vulnerabilidad técnica y legal que la cobija.

Hace pocos lustros, quién hubiera imaginado que los medios electrónicos como internet modificarían los estilos de vida de las personas y que la interconexión entre los seres humanos sería ilimitada, desde diferentes partes del mundo, a través de computadoras y teléfonos.

Esta era digital ha traído tanto grandes beneficios para el progreso de los países, como grandes problemas. No tiene vuelta atrás y lo que se ha conseguido no puede terminar, pues su desarrollo y alcances apenas están asomando su potencial. La denominada tecnología digital "ha creado y criado un nuevo orden mundial en donde mucho de lo que era imposible ahora es posible". ${ }^{12}$

La nueva configuración que las tecnologías de la información y las comunicaciones han propiciado en estos tres primeros lustros del siglo XxI significa un cambio en los conceptos jurídicos tradicionales. La normatividad, paulatinamente, está regulando hechos que suceden en escenarios virtuales y replantea un análisis respecto a lo que se atribuye como espacio público y espacio privado.

\footnotetext{
"Stefik, Mark, The Internet Edge. Social, Technical, and Legal Challenges for a Networked World. The Digital Keyhole: Privacy Rights and Trusted Systems, Estados Unidos, Mit Press, 2000, p. 201.

${ }^{12}$ Craig, Terence y Ludloff, Mary, Privacy and Big Data, Estados Unidos, O'Reilly, 2011, p. 1.
} 
Aunque el término privacidad se utiliza regularmente en la connotación anglosajona como privacy, algunos autores refrendan que para este caso podría fungir también como sinónimo de "intimidad de la vida privada". Pero, al contextualizarse en internet, puede citarse como "derecho a la privacidad en Internet". ${ }^{13}$ En ninguna circunstancia el término deja de ser parte de los denominados derechos de la personalidad. Es decir, todo aquello que concierne a las personas y cuya definición puede ser la siguiente: "Son aquellos que se refieren a las facultades jurídicas cuyo objeto son los diversos aspectos de la propia persona del sujeto, bien como sus emanaciones y prolongaciones". ${ }^{14}$

Tomando en cuenta la definición anterior, y ante cualquier otra que explique este concepto sobre los derechos de la personalidad, Drummond plantea tres sustratos iusfilosóficos paradigmáticos con esta categoría jurídica. En primera instancia está la cosmovisión o "visión del mundo" de cada individuo, pues "gran parte de los bienes jurídicos tutelados por los derechos de la personalidad son bienes que requieren una interpretación subjetiva: el honor, la privacidad y la imagen en cuanto atributo". El segundo es el relativo a "los conflictos entre derechos de la personalidad, como por ejemplo el derecho a la información, especialmente el derecho de informar, y el derecho a la privacidad. Por último, el tercer sustrato, y considerado el más importante, es el relativo al cruce entre el derecho a la privacidad e internet. Aquí, se encuentran los conflictos éticos, bioéticos y el uso propiamente dicho de la red de redes. ${ }^{15}$

Algo que cada ciudadano tiene derecho de decidir sobre su persona e intimidad y en donde nadie más puede intervenir se traduce en la siguiente frase: "Un alejamiento confortable del mundo exterior, por parte del titular del derecho a la privacidad, sólo puede darse o desearse, por el mismo". ${ }^{16}$ Pero, en el ambiente corporativo, el deseo o el derecho a resguardar información para cualquier fin ya no depende solamente del empleado, sino también de quien resguarda su información, cuyo principal responsable es el empleador que la solicitó y se comprometió a cuidarla de cualquier intromisión.

\section{Implicaciones de un derecho a la privacidad en la empresa}

Así como la palabra privacidad remite a la palabra anglosajona privacy y que en la lengua española se ciñe a la "intimidad en la vida privada”, el vocablo

\footnotetext{
${ }^{13}$ Drummond, Victor, Internet, privacidad y datos personales, Madrid, Reus, 2004, p. 32.

${ }^{14}$ Drummond, Victor, Internet, privacidad y datos personales, Madrid, Reus, 2004, p. 35.

${ }^{15}$ Drummond, Victor, Internet, privacidad y datos personales, Madrid, Reus, 2004, p. 36.

${ }^{16}$ Drummond, Victor, Internet, privacidad y datos personales, Madrid, Reus, 2004, p. 37.
} 
intimidad es todavía más cauto pues "se relaciona estrechamente con el ámbito familiar y doméstico, es decir, ajeno al interés público y, por tanto, reservado". ${ }^{17}$ Algunos autores delimitan muy sutilmente ambos términos, otorgando a la privacidad más concesiones. Señalan que la privacidad se refiere al conjunto de actividades de una persona que, sin ser íntimas, requieren del respeto "para garantizar el normal desenvolvimiento y la tranquilidad de los titulares particulares".

La intimidad es todavía más interna, casi psicológica, como lo que está "de la piel del hombre hacia dentro, sentimientos, apetencias, inclinaciones, ideas". ${ }^{18}$ Estos conceptos que, si bien están regulados en casi todas las normativas de los diferentes países, tienen marcadas disparidades reguladoras por el tipo de cultura y costumbres propias de cada entidad.

Los datos o información personal que se proporcionan de manera voluntaria, o a veces involuntaria, pueden ser para mostrarse socialmente en alguna plataforma tecnológica, sobre algún suceso o actividad que se quiera dar a conocer. También puede ser un requerimiento de algún servicio de gobierno o de particulares, como una empresa o negocio. En cualquiera de los casos, la información personal estará disponible en sistemas o soportes electrónicos clasificados por asuntos que convengan a tantos intereses o motivos tenga quien los detenta.

Esas clasificaciones o segmentaciones de la información personal serán determinantes para marcar y atraer los intereses de quienes atenten contra la seguridad de esos datos resguardados, por decir, en lo relativo a edad, sexo, estado civil, ingresos, domicilio, gustos, preferencias y demás aspectos personalísimos, considerados como información altamente vulnerable y que constituye un objetivo central para los delincuentes del ciberespacio.

Los seres humanos gozan de una vida privada, compuesta por todo aquello que no forma parte de actividades catalogadas como públicas, sino aquellas que transcurren en la intimidad de cada persona. En la privacidad no debe haber intromisión de gente externa a la que no le competen los asuntos y actividades que en ese entorno íntimo o familiar ocurren.

Tener privacidad y preservarla forma parte de una serie de garantías individuales a las cuales todo ciudadano tiene derecho. Este es un derecho humano fundamental y, por encima de todo, es mucho más vulnerable cuando se ejerce por medio de la red, que soporta una gran cantidad de datos de seres humanos, de personas comunes, almacenados en diferentes soportes electrónicos.

\footnotetext{
${ }_{17}$ Álvarez Caro, Maria, Derecho al olvido en internet: el nuevo paradigma de la privacidad en la era digital, Madrid, Reus, 2015, p. 27.

${ }_{18}$ Álvarez Caro, Maria, Derecho al olvido en internet: el nuevo paradigma de la privacidad en la era digital, Madrid, Reus, 2015, p. 27 y 28.
} 
La información más vulnerable es la que se muestra al exterior, por medio de videos y fotografias, especialmente cuando se utiliza correo electrónico y redes sociales. Estas últimas son un escaparate de primer impacto que alberga personas conocidas y desconocidas con acceso a las imágenes e información que por ese medio, de manera voluntaria o involuntaria, comparten sus dueños. Curiosamente, algunas personas no le dan importancia al manejo de sus datos en la red y exponen sin cuidado alguno su seguridad personal, la de su familia y la de los propios amigos. Al ser las redes sociales un espacio de entretenimiento, la mayoría desconoce las consecuencias que pueden tener las actividades en ese medio.

Algunos países tienen regulaciones jurídicas donde queda expresa la información personal que se considera íntima. Sin embargo, cada quién valora su información personal para darle el sesgo de privacidad que requiera. Desafortunadamente, al no existir parámetros internacionales y normas eficaces en cuanto a lo que se debe permitir subir o no a las redes, o bien, la restricción de terceras personas que busquen el perjuicio del titular de esa información, las personas usuarias creen que la seguridad informática está garantizada y nadie puede vulnerar sus espacios electrónicos con dolo y mala fe.

La información que se entrega a diferentes organizaciones con propósitos definidos que van desde la solicitud de un servicio hasta la compra o venta de algún artículo, está conformada principalmente por aspectos como nombre, domicilio, teléfono, edad, sexo, escolaridad, estado civil, religión, filiación política, ocupación, amigos, familia, cuentas bancarias, pasatiempos, estado de salud, etcétera. Esta documentación "sensible y accesible por cualquiera" 19 representa una auténtica radiografía personal de un individuo. Se encuentra en los soportes electrónicos de tantos negocios como organizaciones que las hayan elaborado o solicitado previamente y que, por diferentes motivos, "fue entregada de buen agrado y puede ser vista innumerables veces por otros". ${ }^{20}$

Todo documento con información única y personal de cada ciudadano debe ser resguardado y protegido por parte de los particulares que la detenten. Este aspecto, cuya regulación jurídica es propia a la de protección de datos personales, en específico los guardados en bases de datos, soportes o ficheros electrónicos, tiene una íntima relación con el tema de los derechos de la personalidad como el honor, la vida privada, la intimidad y la propia imagen. En todo caso, se trata de

\footnotetext{
${ }^{19}$ Nolan, Catherine y Wilson, Ashley, The Audacity to Spy. How Government, Business and Hackers Rob Us of Privacy, Estados Unidos, Technics Publications, 2014, p. 4.

${ }^{20}$ Nolan, Catherine y Wilson, Ashley, The Audacity to Spy. How Government, Business and Hackers Rob Us of Privacy, Estados Unidos, Technics Publications, 2014, p. 8.
} 
referencias de identidad muy personales cuyo robo y uso indebido atenta directamente, perjudica al individuo y al entorno social que lo rodea.

Esta información personal, que está íntimamente relacionada con aspectos privados de los ciudadanos, incluye la reputación financiera, el estado de salud, y las creencias religiosas, entre muchas más, y se entrega directamente a quien la solicita, por medio de papel o electrónicamente. Las páginas web son un medio rápido y eficaz para recolectar información, pues para tener acceso a algunos portales también se solicita información personal directamente.

Ahora "nuestro mundo ha cambiado; algunos discuten por lo bueno y otros por lo peor. Ahora, nosotros proporcionamos más información sobre nosotros mismos y tenemos más datos colectados y agregados sobre nosotros que cualquier otro grupo en la historia de la humanidad." 21

Orwell, Kafka y Foucault han descrito en sus obras 1984, El proceso y Vigilar y castigar, respectivamente, aspectos relacionados con la violación a la privacidad de los ciudadanos en donde la constante es la intromisión de otros para el control absoluto de sus vidas. Daniel J. Solove, en relación con la obra de Orwell, indica que "Big Brother está constantemente monitoreando y espiando [...] La herramienta primaria de vigilancia es un aparato llamado telescreen el cual es instalado en cada casa y apartamento".

En la obra de Kafka, el personaje es arrestado y acusado, "captura el sentido del desamparo, frustración y vulnerabilidad en experiencias cuando una larga organización burocrática tiene el control sobre una vasta documentación e información de detalles sobre la vida de uno".

Por su parte, Foucault describe que "El Panóptico es un dispositivo de disciplina; es un centro desde el cual se garantiza orden, para prevenir complots y desorden, mandata total obediencia”. Este tipo de vigilancia se utiliza en las cárceles para vigilar a los prisioneros. ${ }^{22}$

Estas obras literarias y filosóficas fueron escritas en diferentes épocas, pero coinciden en la ausencia de tecnología o herramientas avanzadas que podrían haber configurado lo que metafóricamente se menciona en ellas. Sin embargo, hoy, la realidad es diferente y esos supuestos de la literatura de ficción son parte de la realidad actual.

\footnotetext{
${ }^{21}$ Craig, Terence y Ludloff, Mary, Privacy and Big Data, Estados Unidos, O'Reilly, 2011, p. 1.

22 Solove, Daniel, The Digital Person. Technology and Privacy in the Information Age, Estados Unidos, New York University Press, 2004, pp. 29, 30 y 38.
} 


\section{Protección de la información y resguardo de datos en la empresa}

El reconocimiento internacional como derechos humanos que tienen los datos personales los coloca en un plano prioritario para su protección y respeto por parte de las autoridades. No obstante, en la práctica, se demuestra que su vulnerabilidad no radica esencialmente en la esfera pública, en donde las autoridades disponen de la información personal de los ciudadanos, almacenada en bases de datos.

El problema va más allá de la parte gubernamental y se está focalizando en la información o datos personales que obran en manos de particulares y cuyo tratamiento no está siendo adecuado. En este sentido, la información personal, personalísima en varios casos, es de suma importancia para las operaciones cotidianas de distintas empresas. Esto requiere de un manejo y un almacenamiento seguro, y la certeza jurídica necesaria contra un mal uso de la misma.

La Ley Federal de Protección de Datos Personales en Posesión de Particulares (LFPDPPP) de 2010, en su artículo primero menciona que "es de orden público y de observancia general en toda la República y tiene por objeto la protección de los datos personales en posesión de los particulares, con la finalidad de regular su tratamiento legítimo, controlado e informado, a efecto de garantizar la privacidad y el derecho a la autodeterminación informativa de las personas". ${ }^{23}$

Los datos personales, como se ha mencionado, implican cualquier información relacionada con una persona física, sea identificada o identificable, ante la cual existe una obligación de resguardo por parte de sus tenedores y la protección para evitar su divulgación o un mal uso de ella. En el contexto empresarial, toda organización mercantil que realice actos de comercio, cualquiera de los mencionados en las veinticinco fracciones del artículo 75 del Código de Comercio, abre también las posibilidades que se deriven del comercio electrónico y los mensajes de datos, por medio de la tecnología. ${ }^{24}$

Entre la información de carácter personal que es se utiliza para efectos mercantiles, el propio Código de Comercio manifiesta en su artículo 49 que:

Los comerciantes están obligados a conservar por un plazo mínimo de diez años los originales de aquellas cartas, telegramas, mensajes de datos o cualesquiera otros documentos en que se consignen contratos, convenios o compromisos que den nacimiento a derechos y obligaciones.

\footnotetext{
23 "Ley Federal de Protección de Datos Personales en Posesión de Particulares, México", Diario Oficial de la Federación. [Consulta: 4 de abril, 2017]. Disponible en: http://www.diputados.gob.mx/LeyesBiblio/index.htm

24 "Código de Comercio", Diario Oficial de la Federación. [Consulta: 8 de mayo, 2017]. Disponible en: http://www. diputados.gob.mx/LeyesBiblio/index.htm
} 
Para efectos de la conservación o presentación de originales, en el caso de mensajes de datos, se requerirá que la información se haya mantenido íntegra e inalterada a partir del momento en que se generó por primera vez en su forma definitiva y sea accesible para su ulterior consulta. La Secretaría de Economía emitirá la Norma Oficial Mexicana que establezca los requisitos que deberán observarse para la conservación de mensajes de datos.

En este sentido, los datos derivados de un acto de comercio electrónico estarán en soportes ad hoc por un tiempo, durante el cual su tenedor o tenedores, en este caso los empresarios o comerciantes, habrán de cuidarlos ante posibles amenazas que legalmente puedan divulgarlos. Este precepto incluye una interesante variable, dentro de las que se comparten en las transacciones comerciales, como lo es el respaldo de una Norma Oficial Mexicana (Nom), para su conservación. Esta norma, Nom-151-scFI-2016, describe los requisitos que deben observarse para la conservación de mensajes de datos y digitalización de documentos, y concuerda con el citado Código de Comercio. ${ }^{25}$

Si bien, la conservación de datos y documentación relativa a objetivos netamente comerciales tiene un precepto que asegura la información derivada de operaciones mercantiles electrónicas, también funge para aquellas operaciones o actos de comercio tradicionales y que el artículo 38 describe de la siguiente manera:

El comerciante deberá conservar, debidamente archivados, los comprobantes originales de sus operaciones, en formato impreso, o en medios electrónicos, ópticos o de cualquier otra tecnología, siempre y cuando, en estos últimos medios, se observe lo establecido en la norma oficial mexicana sobre digitalización y conservación de mensajes de datos que para tal efecto emita la Secretaría, de tal manera que puedan relacionarse con dichas operaciones y con el registro que de ellas se haga, y deberá conservarlos por un plazo mínimo de diez años. ${ }^{26}$

La anterior disposición se complementa con los subsecuentes artículos 46 y 46 bis. El primero de ellos indica que “Todo comerciante está obligado a conservar

\footnotetext{
25 "Norma Oficial Mexicana, NOM-151-SCFI-2016", Diario Oficial de la Federación. [Consulta: 8 de mayo, 2017]. Disponible en: dof.gob.mx/nota_to_doc.php?codnota $=5478024$

26 "Código de Comercio", Diario Oficial de la Federación. [Consulta: 8 de mayo, 2017]. Disponible en: http://www. diputados.gob.mx/LeyesBiblio/index.htm
} 
los libros, registros y documentos de su negocio por un plazo mínimo de diez años. Los herederos de un comerciante tienen la misma obligación”.

El segundo artículo indica que "Los comerciantes podrán realizar la conservación o digitalización de toda o parte de la documentación relacionada con sus negocios, en formato impreso, o en medios electrónicos, ópticos o de cualquier otra tecnología, siempre y cuando, en estos últimos medios, se observe lo establecido en la norma oficial mexicana sobre digitalización y conservación de mensajes de datos que para tal efecto emita la Secretaría". ${ }^{27}$

Entre las responsabilidades elementales que tiene la empresa en relación con la privacidad y el resguardo de datos de sus empleados, clientes y proveedores, están su cuidado y protección ante cualquier intromisión interna y externa. Esto implica tener en la organización ficheros físicos o electrónicos técnicamente seguros, pero que también sean operados por personal calificado en su manejo y sean éticamente confiables. A pesar de que la información personal que detentan las empresas suele estar amenazada por agentes externos, las amenazas pueden estar más en su interior.

Esa protección a la privacidad de la información en posesión de organismos o empresas, que debe estar reforzada ante cualquier tipo de intromisión, no siempre es importante para los tenedores, ya sea por ignorancia o descuido. Lo peor de esto es que también sucede con el titular o dueño, que ignora o minimiza el uso que ilegalmente se le puede dar a sus datos. A esto se le puede sumar que cada quién marca los límites o grados de su privacidad.

En todo caso, para bien del ciudadano, la legislación que existe garantiza en buena medida el derecho a la privacidad y la protección de datos personales. Existe un derecho humano relativo a la vida privada o a la intimidad con el referente constitucional mexicano del artículo 16, primer párrafo, que indica: "Nadie puede ser molestado en su persona, familia, domicilio, papeles o posesiones, sino en virtud de mandamiento escrito de la autoridad competente, que funde y motive la causa legal del procedimiento". ${ }^{28}$

Este artículo no tiene todavía una legislación secundaria que permita regular todo lo relativo a los derechos de la personalidad, pero es un precepto que ampara en bastantes demandas interpuestas los reclamos de protección de garantías individuales vulneradas a los particulares por actos de autoridad y que perjudican al ciudadano directamente en su persona. En su segundo párrafo, relativo a los datos personales, este mismo artículo constitucional prescribe lo siguiente:

\footnotetext{
27 "Código de Comercio", Diario Oficial de la Federación. [Consulta: 8 de mayo, 2017]. Disponible en: http://www. diputados.gob.mx/LeyesBiblio/index.htm

28 "Constitución Política de los Estados Unidos Mexicanos", Diario Oficial de la Federación. [Consulta: 24 de abril, 2017]. Disponible en: www.diputados.gob.mx/LeyesBiblio/index.htm
} 
Toda persona tiene derecho a la protección de sus datos personales, al acceso, rectificación y cancelación de los mismos, así como a manifestar su oposición, en los términos que fije la ley, la cual establecerá los supuestos de excepción a los principios que rijan el tratamiento de datos, por razones de seguridad nacional, disposiciones de orden público, seguridad y salud públicas o para proteger los derechos de terceros. ${ }^{29}$

En buena parte, el artículo 16 constitucional confirma el tema que ocupa a este apartado, relacionado con la vida privada y la protección de los datos personales de los ciudadanos. Sin embargo, no regula formalmente todo lo que como garantía individual debe tener el ciudadano en estos aspectos, pues todavía no existe una reglamentación total y precisa en la Constitución, o en sus leyes secundarias. No obstante, lo que señala en su párrafo doce concierne de alguna forma:

Las comunicaciones privadas son inviolables. La ley sancionará penalmente cualquier acto que atente contra la libertad y privacía de las mismas, excepto cuando sean aportadas de forma voluntaria por alguno de los particulares que participen en ellas. El juez valorará el alcance de éstas, siempre y cuando contengan información relacionada con la comisión de un delito. En ningún caso se admitirán comunicaciones que violen el deber de confidencialidad que establezca la ley. ${ }^{30}$

Los derechos de la personalidad, cuya base legal se encuentra en la Declaración Universal de los derechos Humanos de 1948, son inherentes al ciudadano y su regulación todavía está en proceso de consolidación. Tienen su fundamentación constitucional en los artículos 6,7 y 16, de los cuales el 6 corresponde a la libertad de expresión y regula también el acceso a la información pública; el 7 es relativo a la libertad de expresión cuyos límites son el respeto a los derechos de terceros, y el 16, antes explicado, versa entre otros aspectos sobre la inviolabilidad de las comunicaciones privadas y de la correspondencia de las personas.

Estos derechos condensan las garantías que tiene todo ciudadano a no ser molestado por autoridades u otras personas en los derechos o prerrogativas que

\footnotetext{
29 "Constitución Política de los Estados Unidos Mexicanos", Diario Oficial de la Federación. [Consulta: 24 de abril, 2017]. Disponible en: www.diputados.gob.mx/LeyesBiblio/index.htm

30 "Constitución Política de los Estados Unidos Mexicanos", Diario Oficial de la Federación. [Consulta: 24 de abril, 2017]. Disponible en: www.diputados.gob.mx/LeyesBiblio/index.htm
} 
posee en su esfera privada. Así, legalmente, puede decidir mantenerlos fuera de lo considerado como público.

En Europa, el Convenio para la Protección de los Derechos Humanos y de las Libertades Fundamentales, firmado en Roma el 4 de noviembre de 1950 y publicado en España en el Boletín Oficial del Estado (вов) número 243 de fecha 10 de octubre de 1979, en su artículo 8, sobre el derecho al respeto a la vida privada y familiar, contiene un par de párrafos que garantizan derechos similares a los mencionados en la legislación mexicana. Dicho precepto, prescribe lo siguiente:

Toda persona tiene derecho al respeto de su vida privada y familiar, de su domicilio y de su correspondencia [...] No podrá haber injerencia de la autoridad pública en el ejercicio de este derecho, sino en tanto esta injerencia esté prevista por la ley y constituya una medida que, en una sociedad democrática, sea necesaria para la seguridad nacional, la seguridad pública, el bienestar económico del país, la defensa del orden y la prevención del delito, la protección de la salud o de la moral, o la protección de los derechos y las libertades de los demás. ${ }^{31}$

Ante el impacto que la tecnología tiene en el derecho, en general, y en los derechos humanos, en particular, la urgencia de nuevos marcos disciplinares es una demanda colectiva en la mayor parte de los países democráticos del mundo. Esto debido a que existen disparidades en el tema de las garantías ciudadanas en ciertas regiones del planeta.

Estas bruscas metamorfosis en el ámbito de las telecomunicaciones no dejan de fascinar a quienes están mundialmente interconectados, disfrutando de los servicios de la moderna sociedad de la información. Pero tampoco dejan de sorprender a los estudiosos del derecho, quienes no alcanzan a encontrar soluciones jurídicas previsoras y precisas para esta cambiante realidad.

Los derechos fundamentales del ciudadano se ven trastocados en esta maraña virtual que redimensiona "las relaciones del hombre con los demás hombres, las relaciones entre el hombre y la naturaleza, así como las relaciones del ser humano para consigo mismo". ${ }^{32}$

\footnotetext{
31 "Convenio para la Protección de los Derechos Humanos y de las Libertades Fundamentales, Roma 1950", Derechos Humanos. [Consulta: 20 de febrero, 2017]. Disponible en: http://www.derechoshumanos.net/Convenio-Europeo-de-Derechos-Humanos-CEDH/index.htm

32 Pérez luño, Antonio Enrioue, Nuevas tecnologías y derechos humanos, Valencia, Tirant lo Blanch, 2014, p. 17.
} 
En este ámbito, también las relaciones laborales entre la empresa y sus colaboradores marcan pautas para determinar los límites entre la preservación y la disposición de la información, así como datos personales que detenta la organización. Ante el mal uso que haga el titular o el tenedor de los mismos, se espera que la propia garantía jurídica contenida en la legislación nacional sea aplicable a cada caso particular.

\section{Conclusiones}

La protección de la privacidad y datos personales de las personas en la sociedad de la información es un tema que compete a todo tipo de ciudadano. Los datos que se obtienen en los servicios de la sociedad de la información están en manos de tantas personas como actividades se realizan. Las empresas tienen una serie de obligaciones que rebasan el simple cuidado de la información y datos personales de sus empleados, clientes y proveedores. Van más allá incluso de lo que prescriben las leyes en asuntos de carácter mercantil.

La preservación de diferentes documentos en papel o en formatos electrónicos requiere el cuidado y la diligencia de quienes los utilizan y disponen de ellos. La privacidad, tema que en sí mismo constituye un derecho de la personalidad, expande el ámbito de su protección cuando se trata del manejo de información o datos personales, entre los cuales puede estar el estatus particular de los ciudadanos, por el respeto que debe imperar en los medios electrónicos hacia los aspectos más reservados y propios de los seres humanos.

Internet es un recurso electrónico, informático y telemático, catalogado como derecho humano, y está plenamente reconocido en las esferas legales de la mayoría de los países del mundo. Su uso no se concreta solamente al goce de una garantía ciudadana que respalda su libre acceso, sino que, al ser un escaparate para colocar información y exponer ideas con plena libertad, este derecho puede exceder los límites y atentar contra los derechos de privacidad y protección de datos personales de los usuarios.

Así, el análisis de esta temática plantea dos interrogantes clave: hasta dónde puede existir la libertad en internet para interactuar, intimar y acceder a la privacidad de los otros, sin previo consentimiento, y hasta dónde llega la responsabilidad de los tenedores de datos personales de los titulares que se encuentren bajo un contrato laboral en alguna corporación o empresa.

La respuesta para ambos cuestionamientos se reduce a la palabra comunicar. Una comunicación masiva, continua y sistemática por todo tipo de medios debe llegar al público en general para informarle y advertirle que en su estatus de empleado, en lo particular, existen riesgos sobre su información y 
documentación personal entregada con fines laborales a un patrón. Este punto es jurídicamente vulnerable para el empresario y también para sus colaboradores, pues ambos requieren estar informados sobre los derechos y obligaciones que existen en esta materia. Asimismo, deben ser cautelosos en el manejo de todo tipo de información contenida en soportes físicos o electrónicos.

\section{Bibliografía}

Álvarez Caro, María, Derecho al olvido en internet: el nuevo paradigma de la privacidad en la era digital, Madrid, Reus, 2015.

Claypoole, Ted y Payton, Theresa, Privacy in the Age of Big Data. Recognizing Threats, Defending Your Rights, and Protecting Your Family, Reino Unido, Rowman Et Littlefield, 2014.

“Código de Comercio", Diario Oficial de la Federación. [Consulta: 8 de mayo, 2017]. Disponible en: http://www.diputados.gob.mx/LeyesBiblio/index.htm

“Constitución Política de los Estados Unidos Mexicanos", Diario Oficial de la Federación. [Consulta: 24 de abril, 2017]. Disponible en: www.diputados.gob.mx/ LeyesBiblio/index.htm

"Convenio para la Protección de los Derechos Humanos y de las Libertades Fundamentales, Roma 1950", Derechos Humanos. [Consulta: 20 de febrero, 2017]. Disponible en: http://www.derechoshumanos.net/Convenio-Europeo-de-Derechos-Humanos-CEDH/index.htm

Cortina, Adela, Construir confianza. Ética de la empresa en la sociedad de la información y las comunicaciones, Madrid, Trotta, 2003.

Craig, Terence y Ludloff, Mary, Privacy and Big Data, Estados Unidos, O'Reilly, 2011.

DRummond, Victor, Internet, privacidad y datos personales, Madrid, Reus, 2004.

"Electronic Communication Privacy Act (ЕсРА)", U. S. Department of Justice, 2016. [Consulta: 27 de marzo, 2017]. Disponible en: https://it.ojp.gov/privacyliberty/authorities/statutes/1285

"Ley Federal de Protección de Datos Personales en Posesión de Particulares, México", Diario Oficial de la Federación. [Consulta: 4 de abril, 2017]. Disponible en: http://www.diputados.gob.mx/LeyesBiblio/index.htm

Nolan, Catherine y Wilson, Ashley, The Audacity to Spy. How Government, Business and Hackers Rob Us of Privacy, Estados Unidos, Technics Publications, 2014.

“Norma Oficial Mexicana, NOM-151-SCFI-2016", Diario Oficial de la Federación. [Consulta: 8 de mayo, 2017]. Disponible en: dof.gob.mx/nota_to_doc.php?codnota $=5478024$ 
Pérez Luño, Antonio Enrique, Nuevas tecnologias y derechos humanos, Valencia, Tirant lo Blanch, 2014.

Pinochet, Francisco, Derecho de Internet. Los principios especiales, México, Flores, 2017.

Solove, Daniel, The Digital Person. Technology and Privacy in the Information Age, Estados Unidos, New York University Press, 2004.

SteFIK, MARK, The Internet Edge. Social, Technical, and Legal Challenges for a Networked World. The Digital Keyhole: Privacy Rights and Trusted Systems, Estados Unidos, MIT Press, 2000.

Warren, Samuel y Brandeis, Louis, “The Right to Privacy”, Harvard Law Review, núm. 193. [Consulta: 20 de febrero, 2017]. Disponible en: http://faculty.uml.edu/ sgallagher/Brandeisprivacy.htm

reseña curricular de la autora:

Licenciada en Derecho por la Escuela Libre de Derecho de Sinaloa. Licenciada en Lengua y Literatura Hispánicas por la Universidad Autónoma de Sinaloa. Maestra en Educación, Especialidad Humanidades, por el Tecnológico de Monterrey. Doctora en Derecho, Sobresaliente Cum Laude, por la Universidad Complutense de Madrid. Profesora investigadora de la Escuela de Ciencias Sociales y Gobierno, del Tecnológico de Monterrey. Sus líneas de investigación son los aspectos legales de la empresa, comercio exterior, internet y comercio electrónico; asimismo, los temas sociales y jurídicos relativos a derechos humanos y seguridad pública. Es autora del libro Aspectos legales del comercio electrónico como comercio transaccional. Es miembro de la American Bar Association (ABA). 